\title{
Challenges in teaching and learning Pediatrics in the present day
}

\author{
Sandra Josefina F. Ellero Grisi ${ }^{1}{ }^{1}$ Ana Maria de Ulhoa Escobar ${ }^{1}$
}

$\mathrm{T}$

The twentieth century was marked by the impressive development of science in all areas. The scientific and technological achievements have enabled mankind to take a giant step towards the cure of diseases and a higher quality of life. The advances in the medical field were many and very important: the elucidation of the human anatomy and physiology; the discovery of the cell, its structure and its biochemical reactions; the development of anesthesia; the elucidation of genetics; the knowledge regarding the immune system; the development of imaging techniques; the discovery of antimicrobial agents and the development of molecular pharmacotherapy, just to mention a few among many others.

The scientific thinking of the twentieth century guided medical reasoning towards the definition of health as a state of absence of illness. And illnesses, in turn, would have their causes primarily determined by a dysfunction of cellular chemistry. The medical researcher of the twentieth century organized the research and deepened his/her knowledge on the structure and function of systems and organs and sought molecular cause-and-effect relationships for many human diseases. Once the cause was found, the study of the pharmacological or surgical cure for the diseases began. As important as the advance of knowledge was the technological advance in this century that ensured the necessary structure for so many important discoveries.

Medical assistance followed the research movement and began assuming a fragmentary thinking pattern organizing themselves in the form of system and even organ specialties. Certainly, this movement had a truly positive impact on the reduction of mortality, on fighting morbidity and therefore on the increase of life expectancy.

This is the so-called biomedical concept of health and illness, according to which "illnesses are well defined entities that involve structural changes at the cellular level and have well-defined causal roots". Thus, the biomedical science model came from the study of organs and systems and their functions and went deeper towards the study of cells and later towards the study of molecules.

In the United States, the teaching of Medicine was based on the Flexner Report, published in 1910, which established strict guidelines for the Medical teaching and practice. According to this report, the modern medical school should be part of a University and have a permanent faculty linked to teaching and research. In turn, the study and knowledge of illnesses, following the biomedical model, became more valued than patient care. As from these guidelines, the medical teaching focused more on hospitals and medical specializations assumed a paramount identity in medical practice, gaining an extraordinary momentum

1. Full Professor of the Department of Pediatrics of the University of São Paulo’s Faculty of Medicine. Email: sandra.grisi@hc.fm.usp.br.

2. Associate Professor of the Department of Pediatrics of the University of São Paulo's Faculty of Medicine. Email: amuescobar@ gmail.com.

Mailing address: Departamento de Pediatria da FMUSP (FMUSP Department of Pediatrics) Av. Dr. Enéas de Carvalho Aguiar, 467. Cerqueira Cesar - São Paulo, SP. ZIP CODE: 05403-000. 
especially from the 40 s on.

The attention of the doctors was transferred from the patients to the illnesses. The diseases started being classified according to a logical system of etiology, physiopathology, clinical picture, diagnosis and therapy. Hospitals came to constitute care, teaching and scientific research centers.

In Faculties of Medicine, teaching in general, to the present day, is guided by the biomedical model. The Medical student, guided by this line of thinking, understands the illnesses as entities that depend on a specific causal model, which has one therapy indicated for all people with a particular cellular and/or genetic disorder. At the same time, during this period, technological development was huge and boosted research and has become a working tool essential to medical practice. The contemporary student, consequently, was taught to value the support medical technology that has become sovereign in clinical decisions.

Thus, if on the one hand the biomedical model unquestionably boosted medical discoveries, which brought fundamental advances in the control of various diseases, not only reducing mortality, but mainly providing better life quality to millions of people, on the other hand, it made human illness be evaluated from the perspective of changes at molecular level. According to this perspective, the human being, as we have seen, was perceived as a fragmented entity likely to be understood and treated by disconnected specialties.

It is imperative to notice that such fragmentation of the individual, when exaggerated, can lead to a blurring of the human condition. The doctors, thus formed, can only focus on human chemistry and biology, at the risk of neglecting psychological, social and environmental aspects of the illnesses.

The teaching of Medicine, from this perspective, has become a challenge for the current times.

A hundred years after the Flexner report, a critical reflection on it points out that the medical teaching, here included the appropriation of contents and the acquisition of skills, should not distance themselves from the ethical and humanistic aspects, essential to the medical practice.

The medical education must therefore be fundamentally structured in human, philosophical and ethical values. This means that the patient's needs must overcome the teaching in all circumstances and the necessary emphasis on the social, economic and cultural aspects of the patient and its social core must be given.

On the other hand, at the beginning of the twentyfirst century, the technological advances in the field of communications became part of everyone's everyday life. Computers, tablets, and cell phones made medical knowledge available at the click of a button, literally on the palm of our hands, and agility and speed were established in the acquisition of medical information. Results from new research are published and read and studied almost in real time, in all regions of the world. The ability to produce knowledge and the access to it promoted a much larger amount of research, exponentially increasing the number of new articles published. The present-day globalized knowledge unifies medical conducts and protocols throughout the world.

The textbooks, as well as the large, heavy and bulky "Medical treaties" lost room on the tables of students. They are replaced by thin electronic devices containing a much greater amount of essential medical content. On the palm of his/her hands, the student can have every chapter in every book needed for their study, daily updated.

The medical schools improved and started to use digital platforms where the program content of every course is made available to students and teachers. More than this: all classes taught by the professors are also included in these platforms, in recorded videos, so that the students can listen and watch them again whenever they can and desire.

As an immediate consequence, the in-house theoretical classes lost room and importance in the virtual universe of the contemporary student.

Perhaps this fact can also explained if we consider that there was, indeed, a significant behavioral change regarding human relationships in the beginning of this century. Social networks enabled a situation where every connected person has a countless number of "friends" with whom they communicate virtually. Therefore, a "new" form of human relationships was created, in which the physical presence is no longer essential. In this perspective, it is considered natural for the professor to also become part of this network of connections where each student, in his or her own available time, turns the professor "on" or "off". Professors appear on the screen teaching their classes in a "click" and disappear instantly in another"click". 
It is understandable that the attention span of the contemporary student has also been modified. The visual stimuli to which they are subjected daily affects their capacity of appropriation of the contents and they have become more sensitive to subjects when they are presented in the form of films, drawings, graphs or self-explanatory illustrations than when they are presented in the form of text.

In this new century, therefore, teachers coming from a non-virtual generation make great efforts to find ways to communicate with students who have already grown up with new learning and behavioral paradigms.

Medical teaching, however, has a peculiarity that makes it supremely unique: the center of the medical attention and action is the human being, with all its plenitude of feelings such as anxiety, fears, afflictions, susceptibilities and insecurity in the face of what is able to threaten his or her life.

In this complex scenario where human relationships occur in a dimension that is not necessarily face-to-face; where professors often teach their lessons in front of a camera; where the technology reigns supreme, defining diagnoses via laboratory tests or very sophisticated imaging, far more valued, even by patients, than the clinical propaedeutics, performed with the ears, eyes and hands of the physician, the challenges to the teaching of medicine are very big.

For the teaching of Pediatrics the challenges are even greater since the doctor-patient relationship involves the intermediation of the family, in which the family and cultural context is relevant and accessible only through personal relationships and where the individual concerned is in the process of being, a condition that requires attention and insight from the physician. The Pediatrics field, nowadays, is quite wide. It begins at the time of conception and continues until the end of adolescence. It is a period of human life characterized by intense plasticity and when emotional experiences will be decisive for the subject's formation. The changes are huge and fast. Pediatricians must be attentive and receptive to information obtained from the family.

Therefore, the teaching of Pediatrics must not, under any circumstances, be limited to the field of pathology, under the penalty of deviating from its most precious and important doctrine: the monitoring of the growth and development of children and adolescents, in all their biological aspects, inserted in their family and community, taking into account their environment and their economic, emotional, psycho-emotional and cultural conditions.

The first 1000 days of life, which includes pregnancy and the first two years of life, are considered to be the "golden" period in which the structures and functions are constructed and established so that the future health and risks for illnesses, as well as cognitive potential and emotional structure, are formed. Pediatricians should be able to understand the context of the family's life and health and along with them design the best childcare proposal. In order to do so, they must be sensitive to family concerns and be able to understand the child's living environment. They need to have an education that goes far beyond biology and technology. The first years of life are absolutely essential and decisive in an individual's life. The plasticity of this period makes the individual very vulnerable to the environment, and therefore, pediatricians must be attentive and stand close to the family. The emotional ties with parents and/or caregivers can interfere with neuronal connections enhancing the cognition capacity and the psychosocial development of children or, on the contrary, disturbing and causing aftereffects in the brain architecture. All the stages that follow, namely pre-school, school and adolescence also have their own specific characteristics which are also essential to human formation. In short, Pediatrics begins with two cells and ends with a formed human being, an adult prepared to face life and all its universe of events.

In this perspective, the teaching of Pediatrics must go beyond the biomedical paradigm and the traditional and updated concepts of the ethio-pathogenic, physiopathological and therapeutic aspects of the acute and chronic illnesses that affect the patient from his/her birth to late adolescence and be able to teach the formation of the individual in the physical, psychological and social level.

For this, the teaching based on classroom practices is necessary. As the teaching of Pediatrics has a substantial part of it occurring in the clinic, it is important to practice values such as human solidarity, respect for the patient and his or her companion, respect for ethnic, religious, gender, cultural or economic differences. 
Medical ethics must necessarily be the most important value on which all technical and human actions are based.

For this reason, the example given by professors and tutors is often the greatest lesson a student can learn. The example does not fit in the Medical Treaties, let alone in digital platforms. The example given by professors is witnessed in person by the students in the everyday medical action where the contact with the patient and his/her family or caregivers are the epicenter of attention. This is and will be the best and most efficient way of teaching medicine and pediatrics: assisting patients with all ethical and human respect and with solid and robust technical knowledge. Technology can be an asset, but not the foundation of teaching. Furthermore, professional responsibility and feelings inherent to the medical practice, such as a spirit of solidarity and compassion can only be learned from human interaction.

This may be the most exciting challenge for the teaching of medicine and pediatrics nowadays, and on every day of all times: to teach the student that the doctor of excellence is above its historical time and must combine, in his or her training, technical knowledge which is available and updated, but also based on humanistic knowledge without ever leaving aside the understanding of others with their most diverse feelings.

The human values are what structures and strengthens the medical practice in its fullness. 